\title{
Comparative evaluation of ultrasonography and computed tomography findings in focal hepatic masses
}

\author{
Bali $\mathbf{S}^{1}$, Kaur $\mathrm{A}^{2}$, Kaur $\mathbf{J}^{3}$, Kumar $A^{4}$, Sachayta ${ }^{5}, \operatorname{Singh~} \mathbf{J}^{6}$ \\ ${ }^{1}$ Dr. Simran Bali, Junior Resident, Radiology, ${ }^{2}$ Dr. Amarjit Kaur, Professor, Radiology, ${ }^{3}$ Dr. Jaswinder Kaur Mohi, \\ Associate Professor, Radiology, ${ }^{4}$ Dr. Ashwani Kumar, Professor, Surgery, ${ }^{5}$ Dr. Sachayta, Senior Resident, Obstetric and \\ Gyaecology, ${ }^{6}$ Dr. Jasvir Singh, Junior Resident, Radiology, all authors are affiliated with GMC Patiala, Punjab, India.
}

Address for Correspondence: Dr. Jasvir Singh, Email: jasvirsingh011789@gmail.com

\begin{abstract}
Introduction: Focal liver lesions are defined as solid or liquid-containing masses foreign to the normal anatomy of the liver that may be told apart from the latter organ using imaging techniques. They can have a number of etiologies including congenital, neoplastic and infectious and inflammatory conditions as well as trauma and miscellaneous entities. The data was analyzed to compare between ultrasonography (USG) and computed tomography (CT) findings in these cases. Aims and Objectives: To evaluate the role of ultrasound and computed tomography in diagnosis of focal hepatic masses and compare the ultrasound and CT findings of focal hepatic masses and correlate with histopathological and surgical findings. Material and Methods: USG and CT were performed on 40 focal hepatic mass patients. The diagnostic value of ultrasound was compared to those of CT. Final diagnosis was made after correlation with surgical findings, serological findings and histopathological examination. The data collected was analyzed statistically. Results: Final diagnosis of focal hepatic masses was simple cysts $(n=5)$, polycystic liver $(n=1)$, metastasis $(n=22)$, hydatid cysts $(n=5)$, hemangioma $(n=6)$, hepatocellular $(n=11)$, focal nodular hyperplasia $(n=1)$, abscess $(n=16)$, cholangiocarcinoma $(n=1)$. The sensitivity, specificity positive and negative likelihood ratio were $84.38 \%, 67.74 \%, 2.62$ and 0.23 respectively, for USG and $100 \%, 97.14 \%, 35$ and 0 respectively, for CT. Conclusion: USG and CT are the modalities having comparable specificity and sensitivity, CT being slightly more accurate than USG in evaluation of focal hepatic lesions in atypical cases. USG is useful in follow-up cases.
\end{abstract}

Key words: Focal hepatic masses, Abscess, Metastasis, Hepatic Cysts.

\section{Introduction}

Detecting and characterization of focal liver lesions is one of the most confusing and controversial challenges in imaging today. A major problem is that all standard non-invasive imaging modalities are less sensitive than generally perceived. These sensitivity problems are no surprise to radiologists experienced in hepatic imaging, since focal hepatic lesions are frequently missed with one modality, then detected with another [1].

\section{Pathologic Classification of Benign Liver Tumors (Based on Cell of Origin)}

Hepatocyte: Hepatocellular adenoma, hepatocellular hyperplasia, focal nodular hyperplasia, macroregenerative nodules, nodular regenerative hyperplasia.

Manuscript received: $14^{\text {th }}$ December 2016

Reviewed: $20^{\text {th }}$ December 2016

Author Corrected: $27^{\text {th }}$ December 2016

Accepted for Publication: $6^{\text {th }}$ January 2017
Bile Duct Epithelium (Cholangiocellular): Hepatic cysts, simple hepatic cysts, polycystic liver disease / congenital hepatic fibrosis

Mesenchymal Cells: Mesenchymalhamartoma, hemangioma, Infantile hemangioendothelioma, lymphangioma.[2]

Hepatocellular Tumors

Primary: Hepatocellular carcinoma (HCC), Fibrolamellar hepatocellular carcinoma, Clear cell carcinoma, Carcinosarcoma, Sclerosing hepatic carcinoma, Hepatoblastoma

Cholangiocellular Tumors: Cholangiocarcinoma, Cystadenocarcinoma

Mesenchymal Tumors: Angiosarcoma, Epithelioid hemangioendothelioma (EHE), Leiomyosarcoma, Malignant fibrous histiocystoma, Lymphoma 


\section{Secondary: Metastatic deposits, Lymphoma [2]}

The choice of imaging test largely depends on the clinical question, availability, the clinicians familiarity with the test and the patient's clinical condition [3]. In addition, access to critical clinical information remains extremely important. The most important clinical parameters defined include age and gender, clinical history, and symptoms [4].

The main goals of imaging are to assess:

- The number and size of the liver abnormalities.

- The location of abnormalities relative to the liver vessels

- The nature of the lesions (benign versus malignant).

- The origin (primary versus secondary) of abnormalities.

- The liver parenchyma surrounding the lesions[5]

In cross sectional imaging, Two basic issues relate to a focal liver lesion: characterization of a known liver lesion and its detection [6]

\section{Imaging Modalities}

Ultrasonography (USG)-Ultrasound is a fundamental technique for imaging the liver, biliary tree and gall bladder because it is inexpensive, easily available and widely accepted. Its advantages are speed and simplicity. Ultrasound is a safe and effective method of detecting focal liver lesions. Its flexibility, easy availability and lack of dependence on organ functions makes it most ideal for imaging the liver and also serves as an object of defining the therapeutic decision quickly. Sonography because of its ability to image in any oblique plane is equal or superior to CT and MRI in localizing lesions to an anatomic segment or subsegment of the liver. Sonography is unexcelled in showing the relationship of liver tumors to critical structures such as veins, bile ducts and arteries.

In addition, sonography can be used for FNAC of these suspicious lesions that might obviate curative hepatic resection.

Apart from detecting lesions, other valuable information like ascites, vessel involvement and primary source of malignancy in abdomen and pelvis can be easily obtained. Being a safe, simple, repeatable and without radiation exposure to the patient it is worthy in being included in routine diagnostic work. Inspite of the advent of the newer diagnostic modalities, it still holds a unique status even in current perspective [7-8].
Computed Tomography- CT offers the best spatial resolution and the ability to study the entire liver in a single breath hold. It serves as an ideal screening examination for the entire abdomen and pelvis. Recent technological advances in CT technology, such as helical CT and multidetector row helical CT have further improved the performance of CT scanners in terms of speed of acquisition, resolution and the ability to image the liver during various phases of contrast enhancement more precisely than was possible previously.

Advances in image post processing and reconstruction methods have enabled the acquisition of three dimensional (3D) images of liver vasculature (CT angiography) to map the liver vascular anatomy and to define the liver and tumour volume. CT is the initial diagnostic test for most indications due to its versatility, availability, high sensitivity and specificity, and the fact that it surveys the entire abdomen for potential metastatic disease in the lymph nodes and peritoneum.

Its limitations include the need for a high radiation dose and a low sensitivity for detection and characterization of lesions smaller than $1 \mathrm{~cm}$. Contrast enhanced CT is contraindicated in patients with a history of anaphylaxis from contrast agents and renal failure [2].

The present study assesses the usefulness of USG and MDCT scan in depicting the status of various focal liver lesions and correlates them.

\section{Material and Methods}

Study Design: This prospective study was done in the Department of Radiodiagnosis, Rajindra Hospital Patiala. 40 patients of hepatic masses were included in this study. All patients presenting with right upper quadrant pain or space occupying lesions of liver i.e. developmental, neoplastic or inflammatory were included in the present study. All traumatic liver lesions were excluded from the study.

Ultrasonography- Ultrasound examination was performed with Philips Envisor or GE Logiq $\alpha-200$ with a 3.5 Mhz sector or curvilinear probes. Acoustic gel was used for skin transducer coupling.

CT scanning- CT examination were performed in all patients on Siemens-Somatom Emotion 6 slice third generation spiral CT. A Triphasic liver CT was performed. The entire liver was scanned successively, 
in arterial, portal and equilibrium phases. Serial CT slices was obtained at a distance of every $8 \mathrm{~mm}$. Patients were given $\mathrm{I} / \mathrm{V}$ contrast of $1.5 \mathrm{ml} / \mathrm{Kg}$ with overall dose ranging from $80-100 \mathrm{ml}$ according to departmental protocol. Patient preparation included administration of $2000 \mathrm{ml}$ of water/gastrograffin 30-60 minutes prior to the examination used as oral contrast. After oral and injection of intravenous contrast material, liver was scanned in arterial (scanning delay, 20-40 seconds), portal (scanning delay, 60-90 seconds), and equilibrium (scanning delay, 2-5 minutes) phases. Enhancement of each lesion in each phase was evaluated, and the lesions were described according to hyper enhancement, hypo enhancement, iso-dense to liver parenchyma and mixed enhancement pattern.

Contrast material- Non-ionic contrast (e.g. iohexol) was used in present study. Contrast was given by peripheral i.v. route and oral route. Continuous monitoring of vital parameters was done during contrast injection. The details of the procedure were explained to the patient. A female attendant was instructed to be present during the procedure in case of female patients. After removing all likely artifacts like pins, chains etc patient was kept supine on CT table which was then advanced into the gantry.

Further the findings were classified as benign or malignant by correlating them with histopathological findings wherever possible. Findings were confirmed either with aspiration and bacteriological analysis, Fine Needle Aspiration Cytology and/or surgery followed by histopathological examination or serology.

Statistical analysis: The data was analyzed statistically. Sensitivity and specificity of ultrasonography and computed tomography were compared.

\section{Results}

This prospective study was done in the Department of Radiodiagnosis, Rajindra Hospital Patiala. In present study there were 24 males and 16 females, with male to female ratio 3:2. 40 patients of hepatic masses were included in this study. Maximum number of patients presented with abdominal pain $(32.5 \%)$ followed by fever in $25 \%$ of cases. Only $2.5 \%$ patients presented with jaundice. $7.5 \%$ of patients were asymptomatic at the time of presentation. The maximum numbers of patients were from age group 51-60 years comprising 25\% of patients followed by patients in the age group of 61-70 years.

Table-1: Types of hepatic masses.

\begin{tabular}{|c|c|c|}
\hline Types of Lesion & No. of Patients & \%age \\
\hline Abscess & 10 & $25.0 \%$ \\
\hline Cholangio Carcinoma & 1 & $2.5 \%$ \\
\hline Focal nodular hyperplasia & 1 & $25.0 \%$ \\
\hline Hepatocellular carcinoma & 10 & $7.5 \%$ \\
\hline Hemangiomas & 3 & $10.0 \%$ \\
\hline Hydatid cysts & 4 & $17.5 \%$ \\
\hline Metastasis & 7 & $2.5 \%$ \\
\hline Polycystic liver & 1 & $7.5 \%$ \\
\hline Simple cysts & 3 & $\mathbf{1 0 0 . 0 \%}$ \\
\hline Total & $\mathbf{4 0}$ & \\
\hline
\end{tabular}

Of the 40 patients studied, maximum cases were of hepatic abscess \& Hepatocellular carcinoma 10 each (25\%) respectively. Metastasis comprised of $17.5 \%$ of the total cases. Minimum number of cases belonged to cholangiocarcinoma, $\mathrm{FNH}$ and polycystic liver $(2.5 \%)$.

Of the 2 cases of amoebic abscesses, one was found to be a single abscess while the other case was of multiple abscesses. The distribution of the lesions was seen in both lobes. The shape varied from irregular shaped to round shaped. The wall appearance varied between irregular and smooth. The wall appeared thick on USG in both amoebic abscess cases. Posterior acoustic enhancement was noted in one case. 
Table-2: USG features of hepatic abscess.

\begin{tabular}{|c|c|c|c|}
\hline & & Amoebic & Pyogenic \\
\hline \multirow[b]{2}{*}{ No. of Abscesses } & Single & 1 & 5 \\
\hline & $\begin{array}{c}\text { Multiple } \\
\text { (More than 1) }\end{array}$ & 1 & 9 \\
\hline \multirow{3}{*}{ Site } & Right Lobe & 1 & 9 \\
\hline & Left Lobe & 1 & 4 \\
\hline & Both Lobe & 0 & 1 \\
\hline \multirow{2}{*}{ Size } & Maximum & $10 \times 8 \mathrm{cms}$ & $11 \times 10$ \\
\hline & Minimum & $3 \mathrm{x} 4 \mathrm{cms}$ & $1.8 \times 1.8$ \\
\hline \multirow{2}{*}{ Shape } & Irregular & 1 & 16 \\
\hline & Round & 1 & 9 \\
\hline \multirow{2}{*}{ Wall Appearance } & Irregular & 2 & 23 \\
\hline & Smooth & 1 & 2 \\
\hline \multirow{2}{*}{ Echotexture } & Hypoechoic & 3 & 13 \\
\hline & Hyperechoic & 0 & 12 \\
\hline \multicolumn{2}{|c|}{ Posterior Acoustic Enhancement } & 1 & 9 \\
\hline
\end{tabular}

In the 8 cases of pyogenic abscesses USG detected 5 single and 9 multiple abscesses. 9 abscesses were detected by USG in the right lobe, whereas 4 were detected in the left lobe, while one abscess was seen to involve part of both left and right lobe. 16 of the pyogenic abscess were irregular in shape while 9 were round in shape.

The wall appearance was found to be irregular in 23 abscesses, while it was found to be smooth in 2 abscesses. Wall thickness was thick in most cases.

13 pyogenic abcesses were found to be hypoechoic while 12 were found to be hyperechoic with internal echoes. 9 pyogenic abscesses showed posterior acoustic enhancement.

Table-3: USG Vs CT imaging features of hepatic abscess.

\begin{tabular}{|c|c|c|c|}
\hline & & USG & CT \\
\hline \multirow{2}{*}{ Wall Thickness } & Thick & 13 & 12 \\
\hline & Thin & 15 & 4 \\
\hline \multirow{2}{*}{ Wall Appearance } & Smooth & 3 & 3 \\
\hline & Irregular & 25 & 13 \\
\hline \multicolumn{2}{|l|}{ Mural Nodule } & 0 & 0 \\
\hline \multicolumn{2}{|l|}{ Internal echoes } & 17 & 0 \\
\hline \multicolumn{2}{|l|}{ Internal Septations / Multiseptated } & 6 & 8 \\
\hline \multicolumn{2}{|l|}{ Air } & 0 & 1 \\
\hline
\end{tabular}

On USG, 16 hepatic abscesses were found to be hypoechoic while 12 hepatic abscesses were found to be hyperechoic.

The walls of 12 abscesses were echogenic. On CT, 4 abscesses showed attenuation more than 20 HU. Also, walls of 16 abscesses showed enhancement. 
Table-4 Comparison of USG and CT in assessment of hepatic lesions.

\begin{tabular}{|c|c|c|c|c|}
\hline Etiology & $\begin{array}{c}\text { USG and CT } \\
\text { Diagnosis }\end{array}$ & USG > Informative & $\begin{array}{c}\text { CT > } \\
\text { Informative }\end{array}$ & $\begin{array}{c}\text { USG \& CT } \\
\text { Indeterminate }\end{array}$ \\
\hline Simple cyst & 5 & - & - & - \\
\hline Hydatid cyst & 5 & - & 1 & - \\
\hline Amoebic Abscess & 3 & - & 11 & - \\
\hline Pyogenic Abscess & 11 & 4 & - & - \\
\hline Polycystic liver & 1 & - & 1 & - \\
\hline Cholangiocarcinoma & - & - & 2 & - \\
\hline HCC & 7 & - & 5 & 1 \\
\hline Metastasis & 14 & - & 4 & - \\
\hline FNH & - & - & - & - \\
\hline Hemangioma & & - & & - \\
\hline
\end{tabular}

Table-5: Final diagnosis with USG.

\begin{tabular}{|c|c|c|}
\hline Statistic & Value & 95\% CI \\
\hline Sensitivity & $84.38 \%$ & $67.21 \%$ to $94.72 \%$ \\
\hline Specificity & $67.74 \%$ & $48.63 \%$ to $83.32 \%$ \\
\hline Positive Likelihood Ratio & 2.62 & 1.54 to 4.45 \\
\hline Negative Likelihood Ratio & 0.23 & 0.10 to 0.53 \\
\hline Disease prevalence & $50.79 \%$ & $37.89 \%$ to $63.62 \%$ \\
\hline Positive Predictive Value & $50.79 \%$ & $55.88 \%$ to $86.21 \%$ \\
\hline Negative Predictive Value & $50.79 \%$ & $60.65 \%$ to $93.45 \%$ \\
\hline
\end{tabular}

Table-6: Final diagnosis with CT.

\begin{tabular}{|c|c|c|}
\hline Statistic & Value & $\mathbf{9 5 \%}$ CI \\
\hline Sensitivity & $100.00 \%$ & $89.42 \%$ to $100.00 \%$ \\
\hline Specificity & $97.14 \%$ & $85.08 \%$ to $99.93 \%$ \\
\hline Positive Likelihood Ratio & 35 & 5.07 to 241.57 \\
\hline Negative Likelihood Ratio & 0 & $0 \%$ \\
\hline Disease prevalence & $48.53 \%$ & $36.22 \%$ to $60.97 \%$ \\
\hline Positive Predictive Value & $97.06 \%$ & $84.67 \%$ to $99.93 \%$ \\
\hline Negative Predictive Value & $100.00 \%$ & $89.72 \%$ to $100.00 \%$ \\
\hline
\end{tabular}

\section{Discussion}

The present study was undertaken to determine the relative role of USG and CT in the diagnosis of focal hepatic masses of liver and also to assess if CT had additional diagnostic value in the detection and characterization of these lesions. The results of imaging were correlated with the final diagnosis, which were obtained by surgery, with histopathology/ aspiration/ follow-up.

In present study, various aetiologies or census of liver lesions were seen. These included simple cysts, polycystic liver disease, liver abscess, hydatid cysts and cystic or necrotic metastasis. The abscess disease was found to be the commonest group seen in 40 patients $(100 \%)$, followed by 7 in metastses and 4 in hydatid cysts. 
Simple Cysts- In present study, simple cysts were seen in 3 cases (7.5\%) with total of 5 cysts, in age group of 3 to 85 years. In present study on USG all the cases (\%age) shows well circumscribed, round to ovoid shaped, anechoic lesions, with smooth margins, without discernible wall with posterior acoustic enhancement and sharp anterior and posterior border. On CT, all 3 cases, were circumscribed, round to oval shaped, with well defined margins, imperceptible wall, homogenous hypo attenuation, with attenuation values $<20 \mathrm{HU}$ and no post contrast enhancement. In a study by Gaines et al [9], 43 cases (100\%) showed that all the simple cysts had no wall abnormalities, internal septation or echoes, no post contrast enhancement.

Hydatid Cyst- In present study, hydatid cysts were seen in 4 cases.

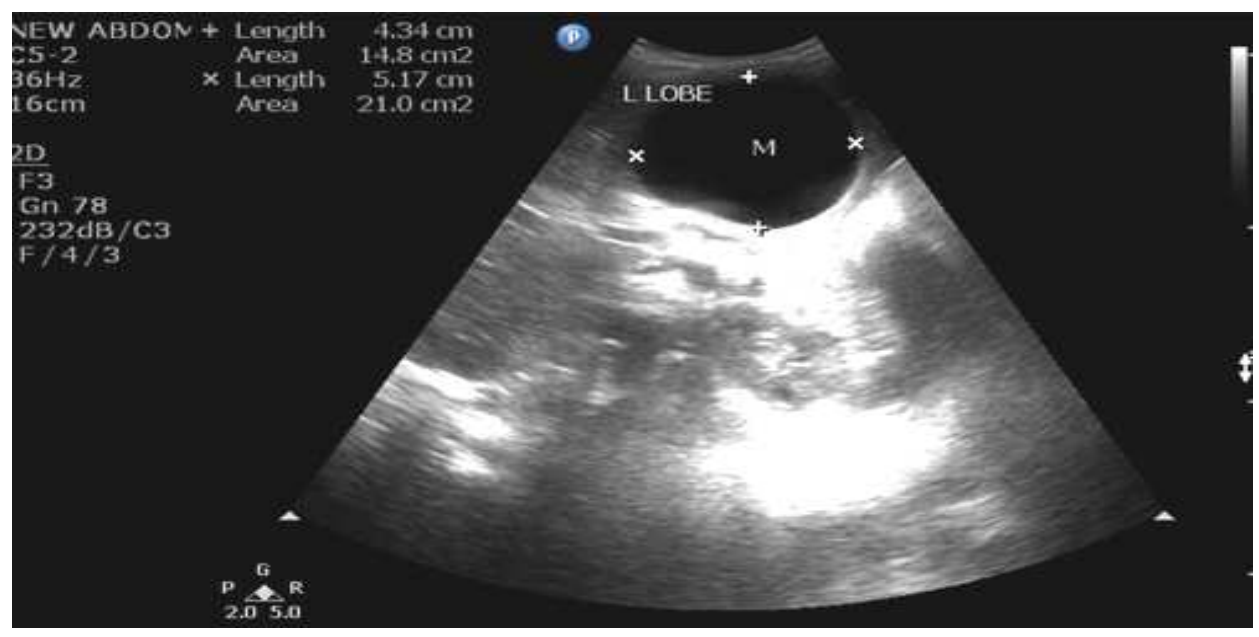

Figure-1 (a): USG image shows thin walled cystic lesion in the left lobe of liver, with posterior acoustic enhancement

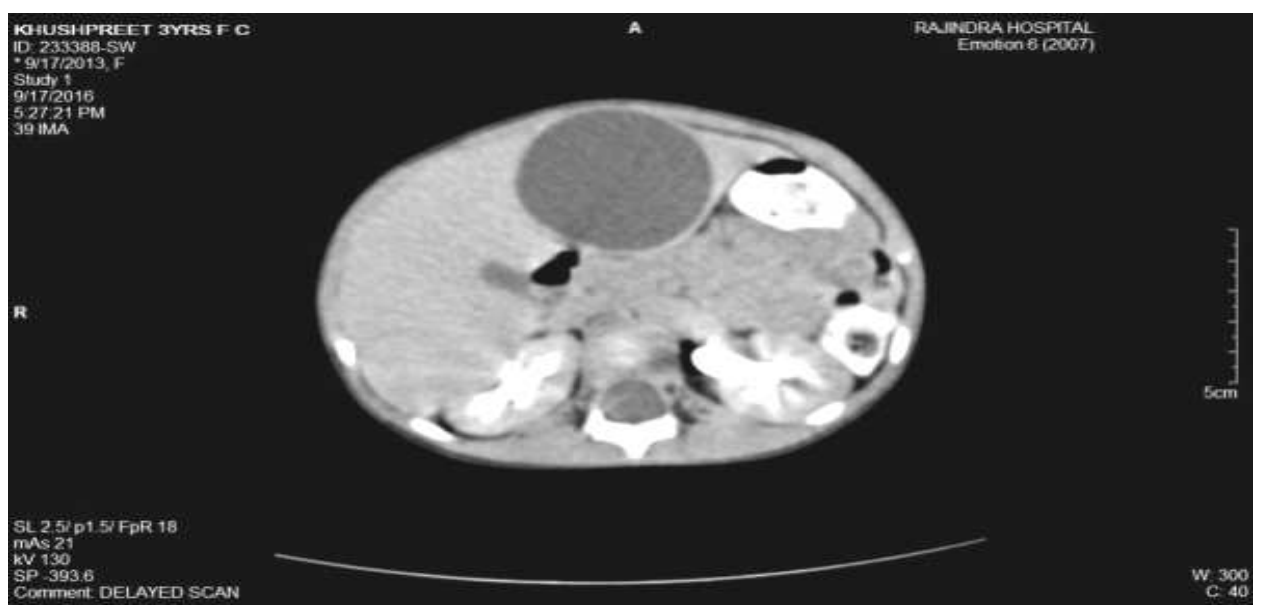

Figure-1 (b): Corresponding CT image shows a large thin walled hypodense lesion of fluid attenuation in the left lobe of liver.

In present study, ultrasonography was $100 \%$ sensitive in detection of hydatid cysts. CT demonstrated cysts with detached membranes in $20 \%$ cases. These detached, free floating membranes, known as water lily sign, which is characteristic and pathognomonic of hydatid cysts.

In a study by Kalinova et al [10] : USG was superior to CT in demonstrating water lily sign and hydatid sand. According to them, other advantages of USG were it is safer, less experience and readily available. In present study, CT demonstrated calcifications in 60\% lesion. USG detected calcifications in only $40 \%$ lesions. CT was superior in demonstrating the calcification. In the studies by El-Tahir et al [11], CT was superior in demonstrating calcification. 
Liver Abscess- In present study there were total 10 cases (25\%) of liver abscesses out of which 7 were males and 3 were females (Figure2). A total of 25 pyogenic lesions were seen on USG, out of which 13 were seen on CT scan. Most common presenting complaint was right upper quadrant pain. The result was comparable with study conducted by Alsaif et al [12] which included patients in the age group of 21-89 years. 94 were males and 37 were females. The most common presenting complaint was fever.

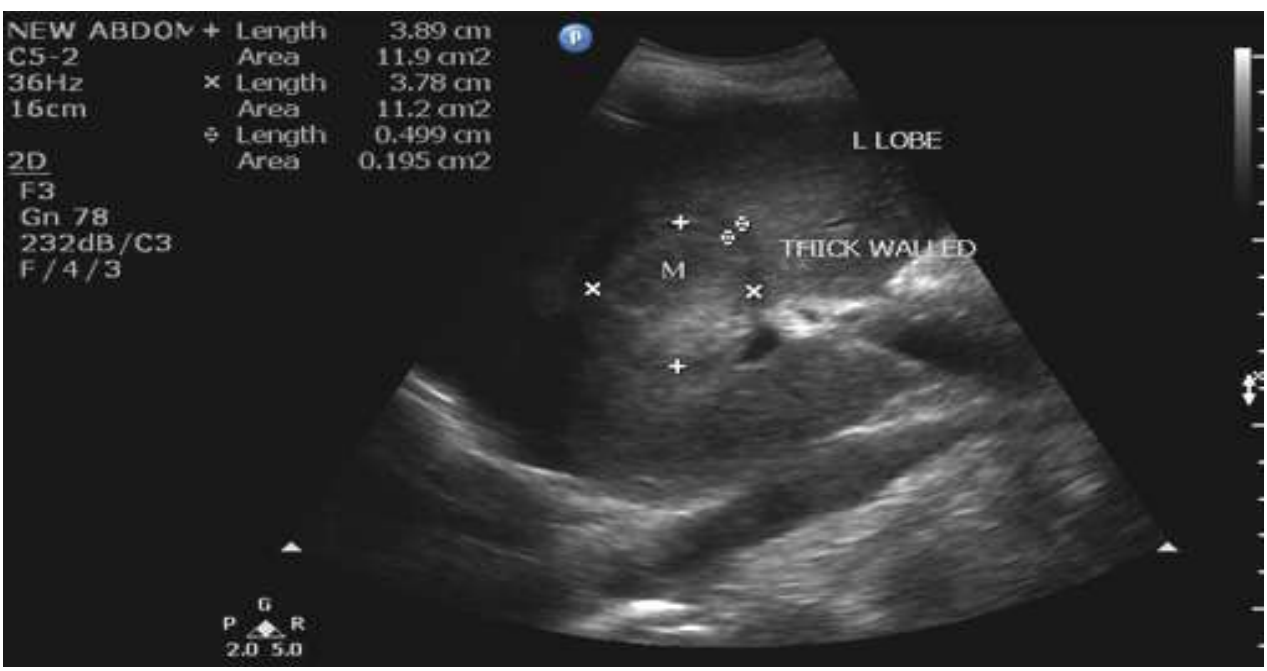

Figure-2 (a): USG showing thick walled hypoechoic lesion with internal echoes and posterior acoustic enhancement

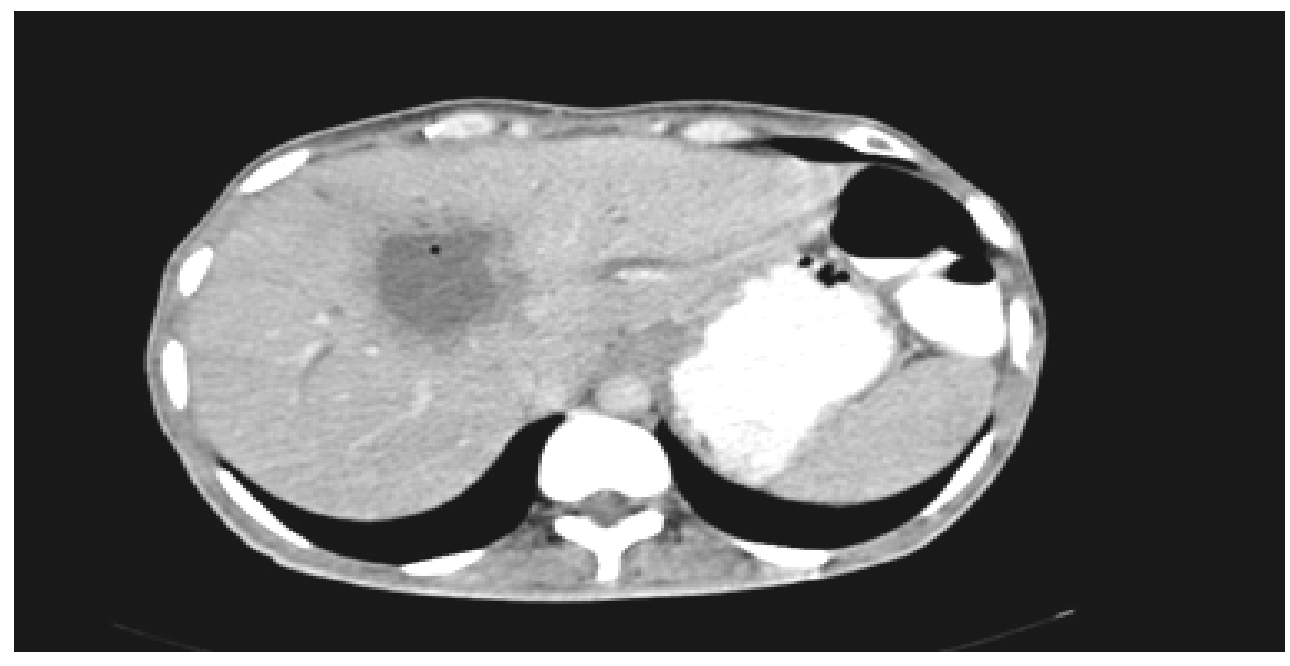

Figure-2 (b): Corresponding CT scan showing a well defined hypodense lesion with a thick enhancing wall and air in the abscess cavity

Metastasis- In present study 65\% of the lesions presented as mixed echo pattern on USG (Figure 3a). In a study conducted by Minami et al [13], the liver is the organ second most commonly affected by metastatic disease. The most common primary sites are the gastrointestinal (GI) tract, lung, breast and head and neck. Therefore, liver metastasis varies in size, shape, vascularity, and growth pattern.

However, most liver metastasis are multiple and show the so-called "cluster sign". In present study 22 lesion of metastasis detected on CT, arterial enhancement was seen in 15 lesions, while delayed enhancement was seen in 1 case. 14 lesions showed enhancement of wall. 15 lesions were found to be hypodense (Figure $3 b$ ), while 2 were found to be hyperdense and 5 showed hetergenous enhancement. Target appearance was seen in 4 lesions. USG incorrectly diagnosed 5 metastatic lesions as pyogenic abscesses. 


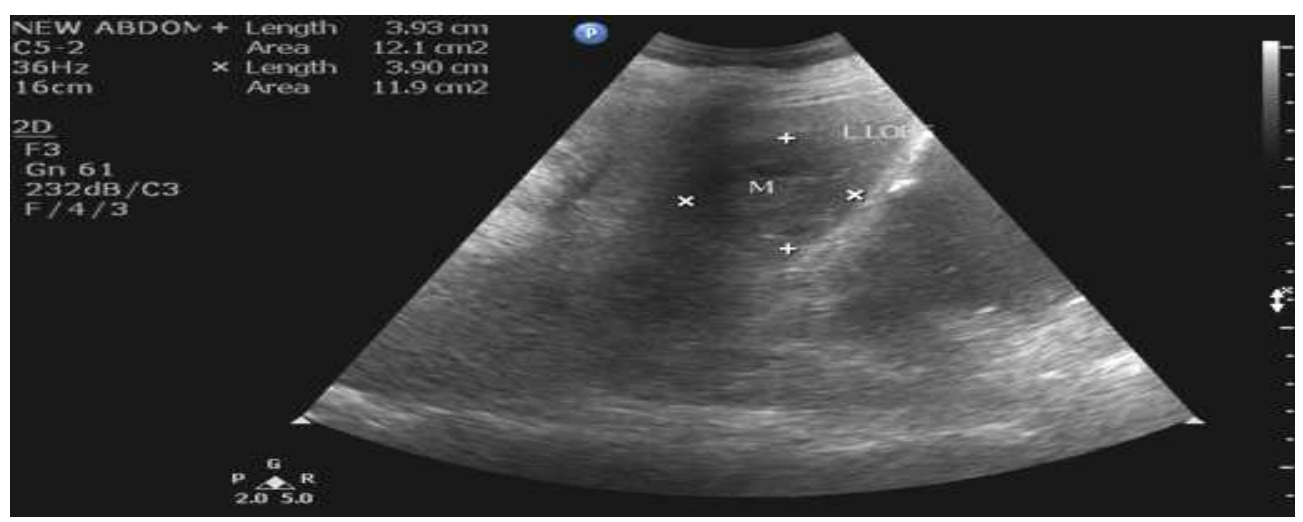

Figure 3(a): USG scan shows a round, heterogenous predominantly hypoechoic lesion with internal echoes, in the left lobe of liver.

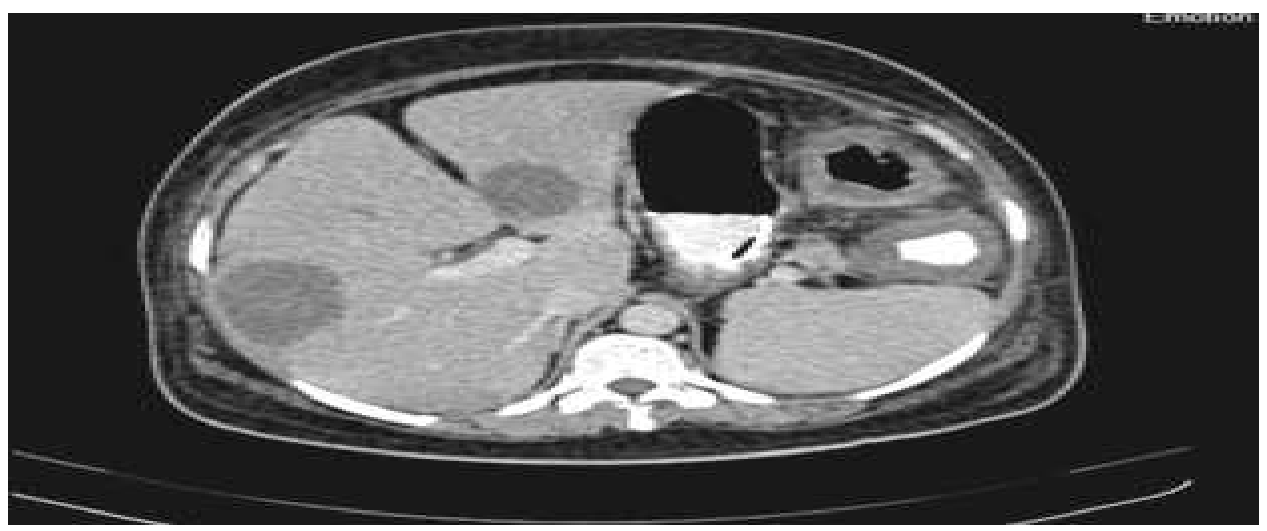

Figure 3(b): Corresponding CT scan shows two well defined peripherally enhancing hypodense lesions in the right and left lobes of liver.

Two lesions diagnosed as metastasis on USG were later found to be haemangiomas on CT. A lesion diagnosed as HCC on both CT and USG was later found to be FNH on histopathological examination.

In a similar study conducted by Sica et al [14], most metastasis are revealed as low- or isoattenuating masses on CT. Depending on lesion size, the margins tend to be irregular, and necrosis may be present, but margins can be sharp and well defined. Central low attenuation may be the result of necrosis or cystic change.

Polycystic disease of liver-In present study 1 case of polycystic liver disease was diagnosed. The patient was asymptomatic. Multiple cysts were seen in both lobes of liver on USG and CT. In a study conducted by Everson et al ${ }^{[15]}$, Polycystic liver disease in genetically linked to protein kinase $\mathrm{C}$ substrate $80 \mathrm{~K}-\mathrm{H}$ (PRKCSH). The cysts are more prominent in women, hepatic cysts emerge after onset of puberty and dramatically increase in number and size through the child-bearing years of early and middle adult life. Although liver failure or complications of advanced liver disease are rare, some patients develop massive hepatic cystic disease and become clinically symptomatic.

Hemangiomas- Hemangiomas are the most common benign tumor of liver. On B-USG, they typically present as homogenously hyperechoic rounded lesions with distinct margins, sometimes with slight posterior acoustic enhancement. In present study 3 cases of hemangiomas with 6 lesions in all were diagnosed with 1 case being of giant hemangioma (Figure4). The lesions on USG varied from mixed to solid appearance with hyperechoic echopattern and shapes varied from irregular to round shape. On CT the lesions were predominantly hypodense in attenuation with irregular to smooth wall appearance. Shapes varied from irregular to round shape. 6 lesions showed enhancement in the arterial and venous phase while 2 lesions were enhancing in the delayed phase as well. In a study conducted by Kumar et al [16] showed sharpely defined highly reflective round tumor larger than $2.5 \mathrm{cms}$ showing posterior acoustic enhancement. CT scan showed typical centripetal type of enhancement. 


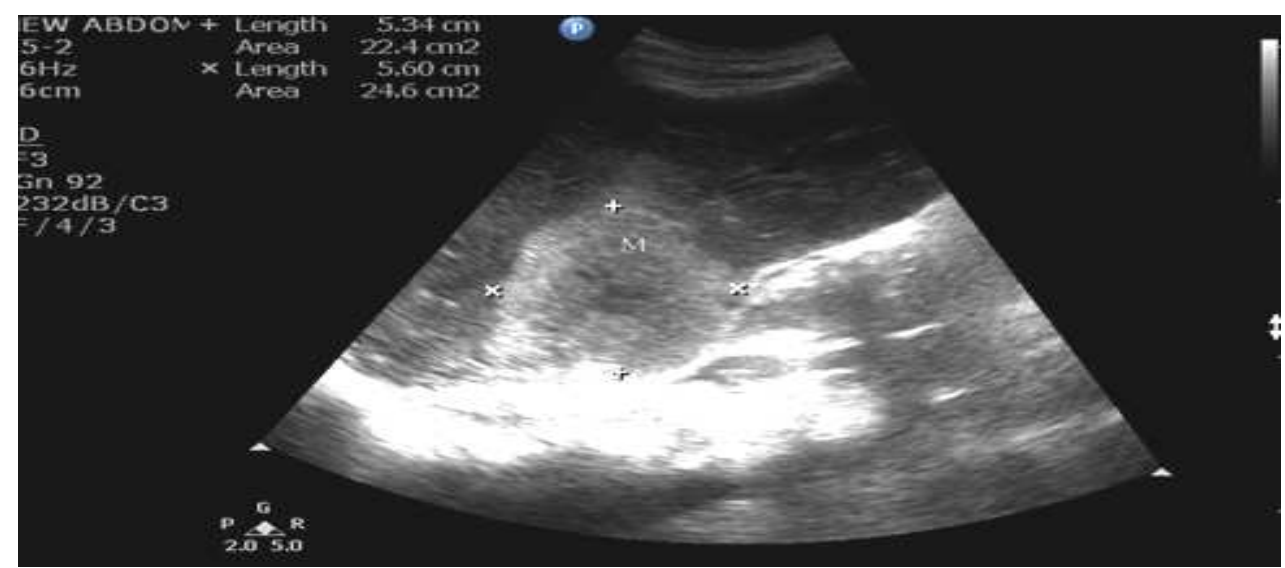

Figure-4 (a): USG shows a rounded hyperechoic lesion in the right lobe of liver

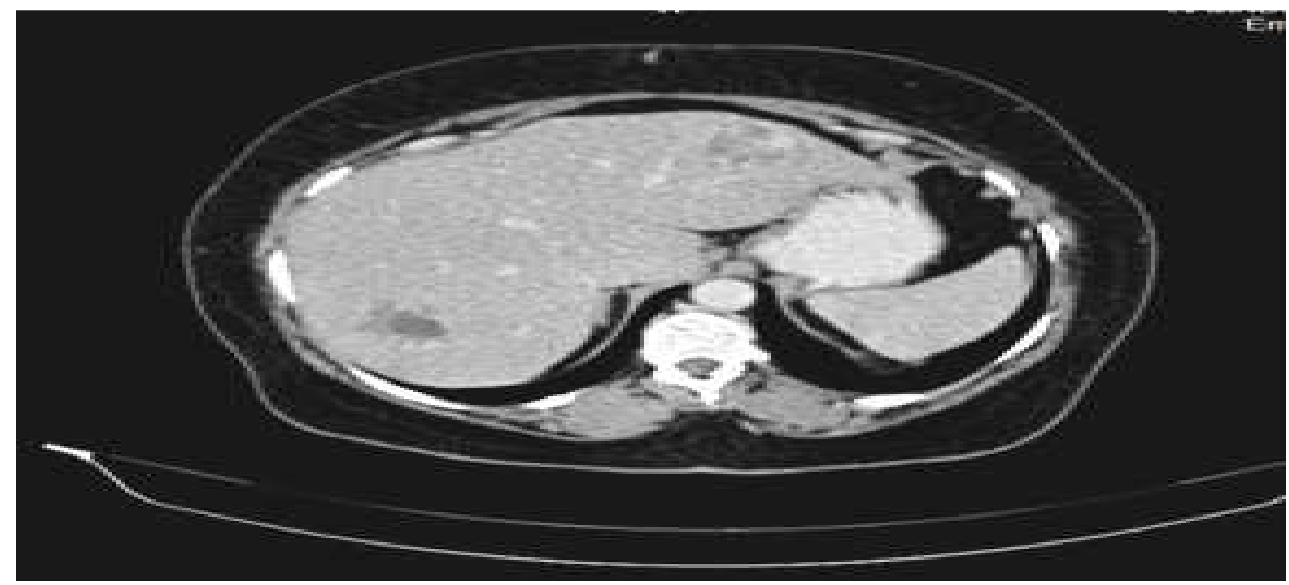

Figure-4(b): Corresponding ct scan shows two rounded hypodense

lesions, one each in the right and left lobe, which show progressive filling in the venous phase.

Focal nodular hyperplasia (FNH)- In a study conducted by D'Onofrio et al[17], they found it is the second most common benign lesion in liver. It cannot be defined as truly neoplastic lesion but rather a regenerative mass of variable size resulting from a vascular abnormality. The typical pathological feature is presence of large central scar in which are artery large them usual is located. In present study, 1 case of FNH (Figure 5) was diagnosed on histopathological examination which ultrasound and CT incorrectly diagnosed as HCC. The lesions was found to be heterogenous predominantly hypoechoic. CT showed an irregularly hyperdense lesion with central hyper attenuating scar.

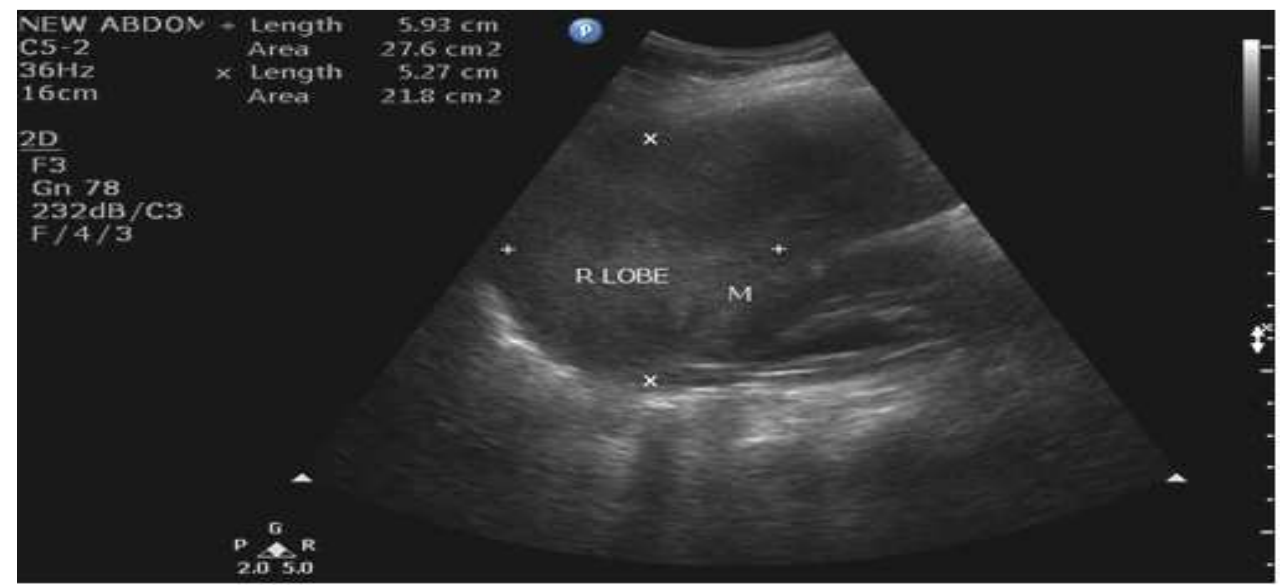

Figure-5 (a): USG shows a heterogenous predominantly hyperechoic mass in the right lobe of liver 


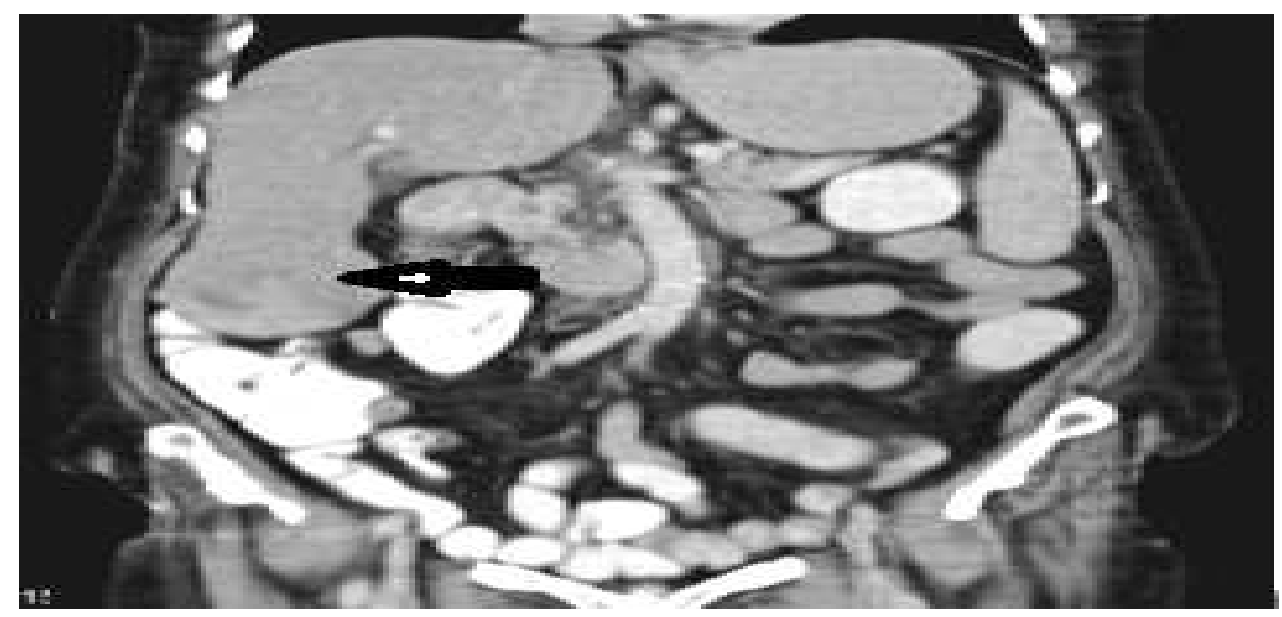

Figure-5 (b) : Corresponding ct image shows an irregularly shaped, heterogenously enhancing area in the right lobe of liver (arrow)

Hepatocellular carcinoma (HCC)-In a study conducted by D'Onofrio et al [17] showed it is the sixth most common neoplasm and most common primary liver malignancy. In most cases, HCC develop victim an established background of chronic liver disease.

USG is most common imaging modality for HCC surveillance in high risk patients because of its efficacy, availability, non-invasiveness and low cost. However, Doppler applied to B-mode USG has low sensitivity in studying blood flow features within a newly discovered lesion.

In present study 9 lesions were diagnosed as HCC on USG (Figure 6a) and 12 lesions on CT (Figure 6b). Out of 9 lesions diagnosed on USG 6 were found to be hyperechoic in echopattern and 3 were hypoechoic, whereas on CT out of the 12 lesions 2 lesions were found hypodense and 8 were heterogeneous in attenuation. On CT 7 lesions showed enhancing walls and 11 lesions showed enhancement on arterial phase. 2 lesions showed delayed enhancement. 3 lesions were found to have echogenic walls on USG. Target appearance was diagnosed on USG and none of the modalities detected any calcification. 6 lesions showed peripheral vascularity on USG while 1 lesion showed internal vascularity.

In a study conducted by Kumar et al [16], 50\% HCC were hypoechoic on USG and 50\% were heterogenous. CT scan showed $83 \%$ HCCs were heterogenous and $17 \%$ hypodense. All lesions showed early heterogenous enhancement in early phases and early washout in delayed phase.

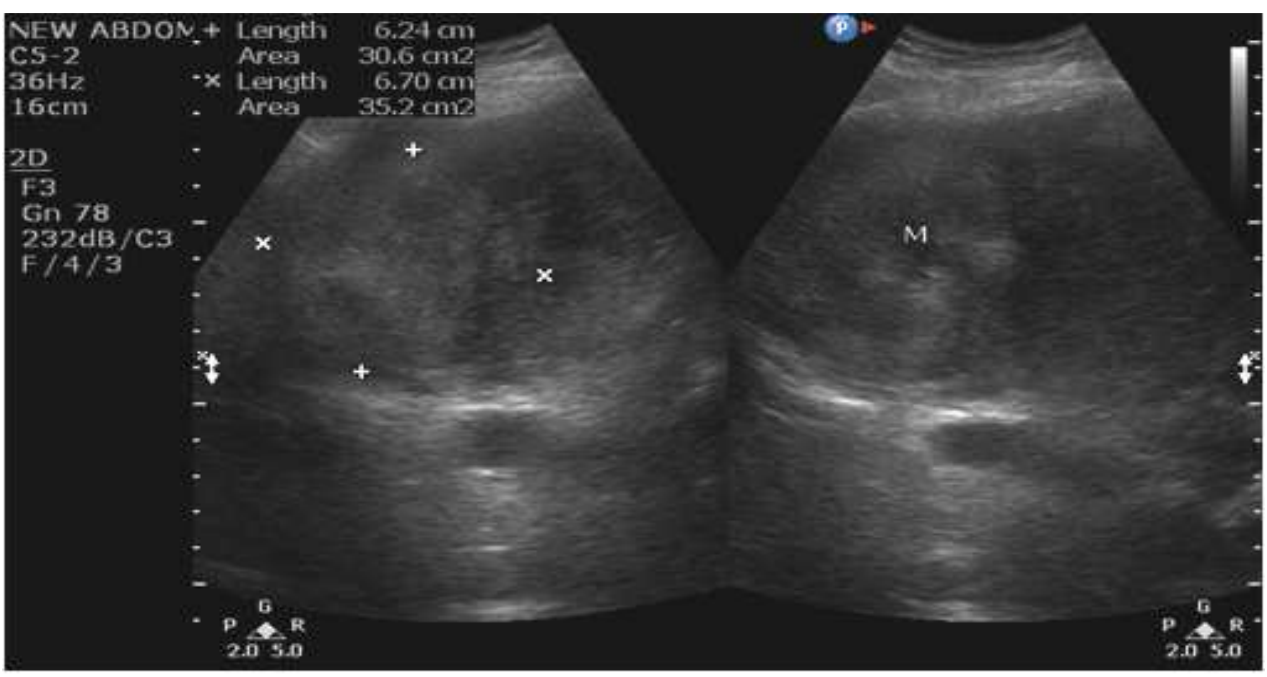

Figure-6 (a): USG image shows a large irregularly shaped hyperechoic mass in the right lobe of liver. 


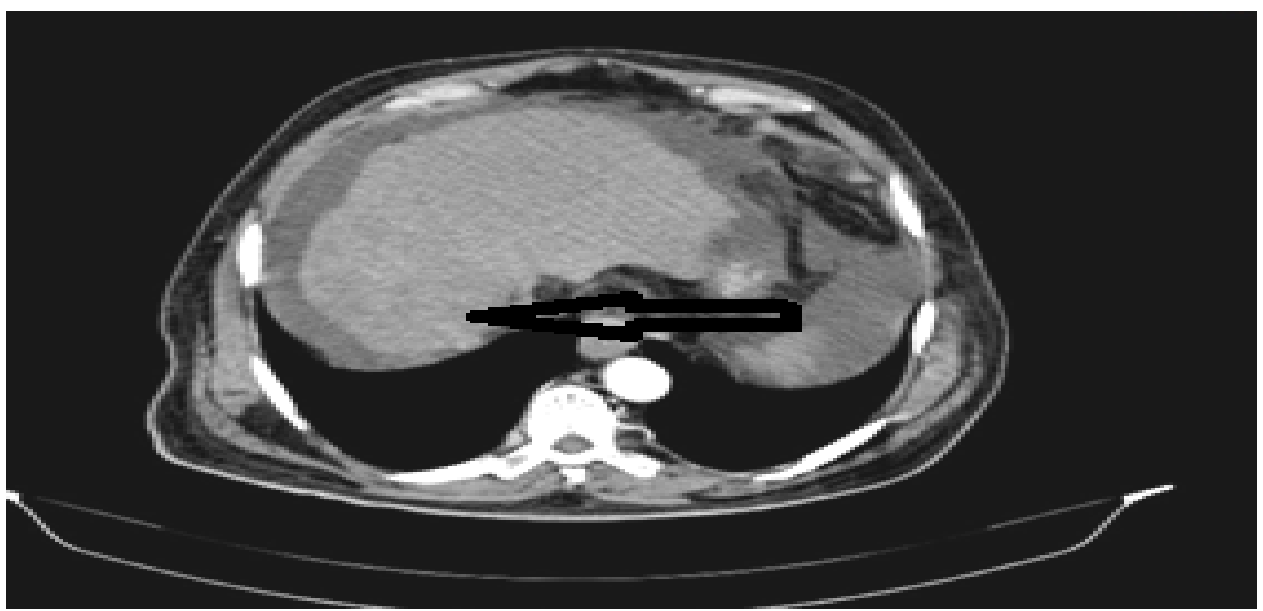

Figure-6 (b): Corresponding ct image shows a large, irregularly shaped hyperdense lesion (indicated by arrows) in the right lobe of liver, with ascites. The mass was found to enhance on arterial phase

Cholangiocarcinoma- D'Onofrio et al [17] found it is the second most common primary liver tumor. Its incidence and modality are increasing because of the late clinical presentation with non specific symptoms and lack of effective nonsurgical therapy. On conventional B-mode USG, it usually appears as an ill defined irregularly hypoechoic mass.In present study a single hypoechoic, irregularly shaped lesion with peripheral vascularity on USG was incorrectly diagnosed as HCC on USG, while the same lesion was seen as having hetergenous attenuation on CT, showing delayed enhancement and was correctly diagnosed as cholangiocarcinoma.

\section{Conclusion}

Liver cysts have typical appearance on USG as well as CT, both the modalities having high sensitivity and specificity. Hence, cysts are diagnosed by one modality further investigation may not be needed. In the case of metastasis, haemangiomas, HCC and cholangiocarcinoma, CT is superior to USG, as these lesions have specific enhancing patterns. Also, CT can accurately show the exact extent of a focal lesion and delineate adjacent organs. Imaging features of amoebic and pyogenic abscesses vary considerably on either modality and require needle aspiration cytology. However, subsequent to treatment, follow up is easier with USG. So in essence, USG and CT are the modalities having comparable specificity and sensitivity, CT being slightly more accurate than USG in evaluation of focal hepatic lesions. In spite of the various advantages of CT over USG, in a developing country like ours, it may be judicious to use ultrasound first because it is widely available, cost effective, non invasive and free from radiation. CT scan may be performed in atypical cases where ultrasound is not confirmatory and to know the exact extent of the lesion prior to surgery. As a follow up modality, in most situations, USG may be adequate.

Funding: Nil, Conflict of interest: None. Permission of IRB: Yes

\section{Bibliography}

1. Tchelepi H, Ralls PW. Ultrasound of focal liver masses. Ultrasound Q. 2004 Dec;20(4):155-69.

2. Gupta AK, Chowdhury V, Khandelwal N. Benign Focal Lesions in Liver. In: Diagnostic Radiology gastrointestinal and hepatobiliary imaging. $3^{\text {rd }}$ edn. Jaypee Brothers Medical Publishers (P) Ltd. 2011; 13:238-45.

3. Sahani DV, Kalva SP. Imaging the liver. Oncologist. 2004;9(4):385-97.

4. Mortelé KJ, Ros PR. Cystic focal liver lesions in the adult: differential CT and MR imaging features. Radiographics. 2001 Jul-Aug;21(4):895-910.

5. Kar $\mathrm{P}$ and Jain R. Imaging of Space Occupying Lesions of Liver. Medicine update 2011:375-84.

6. Thimmaiah VT. Evaluation of focal liver lesions by ultrasound as a prime imaging modality. Sch. J. App. Med.Sci.,2013;1(6):1041-59.

7. Nino-Murcia M, Olcott EW, Jeffrey RB Jr, Lamm RL, Beaulieu CF, Jain KA. Focal liver lesions: pattern- 
based classification scheme for enhancement at arterial phase CT. Radiology. 2000 Jun;215(3):746-51.

8. Sukov RJ, Cohen LJ, Sample WF. Sonography of hepatic amebic abscesses.AJR Am J Roentgenol. 1980 May;134(5):911-5.

9. Gaines PA, Sampson MA. The prevalence and characterization of simple hepatic cysts by ultrasound examination. Br J Radiol. 1989 Apr;62(736):335-7.

10. Kalinova K. Imaging (ultrasonography, computer tomography) of patients with hydatid liver disease. Bulg. J. Vet. Med. 2007;10(1):45-51.

11. el-Tahir MI, Omojola MF, Malatani T, al-Saigh AH, Ogunbiyi OA. Hydatid disease of the liver: evaluation of ultrasound and computed tomography. Br J Radiol. 1992 May;65(773):390-2.

12. Alsaif HS, Venkatesh SK, Chan DS, Archuleta S. CT appearance of pyogenic liver abscesses caused by Klebsiella pneumoniae. Radiology. 2011 Jul;260 (1): 129-38.doi:10.1148/radiol.11101876. Epub 2011 Apr 1.
13. Minami Y, Kudo M. Hepatic malignancies: Correlation between sonographic findings and pathological features. World J Radiol. 2010 Jul 28;2 (7) : 249-56. doi: 10.4329/wjr.v2.i7.249.

14. Sica GT, Ji H, Ros PR. CT and MR imaging of hepatic metastases. AJR Am J Roentgenol. 2000 Mar; 174(3):691-8.

15. Everson GT, Taylor MR, Doctor RB. Polycystic disease of the liver. Hepatology. 2004 Oct;40(4): 774-82.

16. Kumar PB, Hegde P, Kumar BNK, Kumar MLH, Manjunatha YC. A comparative study of ultrasound and CT findings in focal liver lesions. Int Biol Med Res 2014;5(3):4362-69.

17. D'Onofrio M, Crosara S, De Robertis R, Canestrini S, Mucelli RP. Contrast-Enhanced Ultrasound of Focal Liver Lesions. AJR Am J Roentgenol. 2015 Jul;205(1):W56-66. doi: 10.2214/AJR.14.14203.

\section{How to cite this article?}

Bali S, Kaur A, Kaur J, Kumar A, Sachayta, Singh J. Comparative evaluation of ultrasonography and computed tomography findings in focal hepatic masses. Int J Med Res Rev 2017;5(01):03-14.doi:10.17511/ijmrr. 2017.i01.01 